\title{
London penetration depth and coherence length of SU(3) vacuum flux tubes
}

\author{
Paolo Cea \\ Dipartimento di Fisica dell'Università di Bari \\ and INFN - Sezione di Bari, I-70126 Bari, Italy \\ E-mail: paolo.cea@ba.infn.it

\section{Leonardo Cosmai} \\ INFN - Sezione di Bari, I-70126 Bari, Italy \\ E-mail: leonardo.cosmai@ba.infn.it
}

\section{Francesca Cuteri*}

Dipartimento di Fisica dell'Università della Calabria

and INFN - Gruppo collegato di Cosenza, I-87036 Arcavacata di Rende, Cosenza, Italy

E-mail: francesca.cuteri@fis.unical.it

\section{Alessandro Papa}

Dipartimento di Fisica dell'Università della Calabria and INFN - Gruppo collegato di Cosenza, I-87036 Arcavacata di Rende, Cosenza, Italy

E-mail: alessandro.papaefis.unical.it

\begin{abstract}
The transverse profile of the chromoelectric field generated by a quark- antiquark pair in the SU(3) vacuum is analysed within the dual superconductor scenario, then the London penetration depth and coherence length are extracted. The color field is determined on the lattice through a connected correlator of two Polyakov loops measured on smeared configurations.
\end{abstract}

The 32nd International Symposium on Lattice Field Theory,

23-28 June, 2014

Columbia University New York, NY

\footnotetext{
*Speaker.
} 


\section{Introduction}

As is well known, the chromoelectric flux tubes produced by a pair of static color charges in the QCD vacuum represent an evidence for the confinement phenomenon [1]. Monte Carlo simulations of lattice QCD [2-4] allow nonperturbative studies of the chromoelectric field distribution associated with the flux-tube structures. Within the dual superconductor model of QCD vacuum, conjectured by 't Hooft and Mandelstam [5], the condensation of color magnetic monopoles responsible for the formation of flux tubes is seen in analogy to the formation of Cooper pairs in the BCS theory of superconductivity. Whereas the dynamical condensation of color magnetic monopoles is not explained by the dual superconductor construction, convincing lattice evidences for this condensation mechanism have been found [6]. In previous studies [4, 7], the flux-tube chromoelectric field distribution has been investigated through the connected correlation function [3, 8]:

$$
\rho_{W}^{\text {conn }}=\frac{\left\langle\operatorname{tr}\left(W L U_{P} L^{\dagger}\right)\right\rangle}{\langle\operatorname{tr}(W)\rangle}-\frac{1}{N} \frac{\left\langle\operatorname{tr}\left(U_{P}\right) \operatorname{tr}(W)\right\rangle}{\langle\operatorname{tr}(W)\rangle},
$$

where $U_{P}=U_{\mu v}(x)$ is the plaquette in the $(\mu, v)$ plane, connected to the Wilson loop $W$ by a Schwinger line $L$, and $N$ is the number of colors (see Fig. 1 in Refs. [7]). In the naive continuum limit [3] we have

$$
\rho_{W}^{\text {conn }} \stackrel{a \rightarrow 0}{\longrightarrow} a^{2} g\left[\left\langle F_{\mu v}\right\rangle_{q \bar{q}}-\left\langle F_{\mu \nu}\right\rangle_{0}\right], \quad F_{\mu v}(x)=\sqrt{\frac{\beta}{2 N}} \rho_{W}^{\text {conn }}(x) .
$$

where \langle\rangle$_{q \bar{q}}$ denotes the average in the presence of a static $q \bar{q}$ pair and $\langle\quad\rangle_{0}$ is the vacuum average.

In ordinary superconductivity tube-like structures arise as solutions of the Ginzburg-Landau equations [9]. Within dual superconductivity, the formation of the chromoelectric flux tubes can be interpreted as dual Meissner effect and the chromoelectric field distribution should resemble the dual version of the Abrikosov vortex field distribution. This led to the proposal $[4,7]$ to fit the transverse shape of the longitudinal chromoelectric field according to

$$
E_{l}\left(x_{t}\right)=\frac{\Phi}{2 \pi} \mu^{2} K_{0}\left(\mu x_{t}\right), \quad x_{t}>0,
$$

where $K_{n}$ is the modified Bessel function of order $n, \Phi$ is the external flux, and $\lambda=1 / \mu$ is the London penetration length. However, Eq. (1.3) is valid only for type-II superconductors, i.e. for $\lambda \gg \xi, \xi$ being the coherence length, which measures the coherence of the magnetic monopole condensate. Several numerical studies [10] have, instead, shown that the confining vacuum behaves much like a dual superconductor lying on the borderline between type-I and type-II superconductivity. Nonetheless, in Ref. [11] it has been suggested a different fitting function by exploiting the results in Ref. [12]. There, from the assumption of a simple variational model for the magnitude of the normalized order parameter of an isolated vortex, analytic expressions for magnetic field and supercurrent density are derived, that solve the Ampere's law and the Ginzburg-Landau equations. By dual analogy

$$
E_{l}\left(x_{t}\right)=\frac{\phi}{2 \pi} \frac{1}{\lambda \xi_{v}} \frac{K_{0}(R / \lambda)}{K_{1}\left(\xi_{v} / \lambda\right)}, \quad R=\sqrt{x_{t}^{2}+\xi_{v}^{2}},
$$

where $\xi_{v}$ is a variational core-radius parameter. Equation (1.4) is equivalent to

$$
E_{l}\left(x_{t}\right)=\frac{\phi}{2 \pi} \frac{\mu^{2}}{\alpha} \frac{K_{0}\left[\left(\mu^{2} x_{t}^{2}+\alpha^{2}\right)^{1 / 2}\right]}{K_{1}[\alpha]}, \quad \mu=\frac{1}{\lambda}, \quad \frac{1}{\alpha}=\frac{\lambda}{\xi_{v}} .
$$


By fitting Eq. (1.5) to $E_{l}\left(x_{t}\right)$ data, one can extract both the penetration length $\lambda$ and $\lambda / \xi_{v}$. The Ginzburg-Landau $\kappa$ parameter can then be obtained by

$$
\kappa=\frac{\lambda}{\xi}=\frac{\sqrt{2}}{\alpha}\left[1-K_{0}^{2}(\alpha) / K_{1}^{2}(\alpha)\right]^{1 / 2},
$$

and, the coherence length $\xi$ can be deduced.

With the final aim of extending the analysis of flux tubes to the case of finite temperatures (where the study of these structures is directly relevant to clarify the formation of $c \bar{c}$ and $b \bar{b}$ bound states in heavy ion collisions), we cannot employ Eq. (1.1). Nevertheless, it suffices replacing, in Eq. (1.1), the Wilson loop with two Polyakov lines (see Fig. 1). In addition, also the cooling mechanism, previously used to enhance the signal-to-noise ratio, was replaced in our work by the APE smearing procedure [13], to get rid of lattice artifacts. Preliminarily, a check that this method gives results consistent with previous studies, adopting Wilson loops and cooling, is necessary, and that is the subject of the present work (see Ref. [14] for more details). Indeed, numerical results on the chromoelectric flux tubes in $\mathrm{SU}(3)$ pure gauge theory at zero temperature, obtained with connected correlations built with Polyakov lines and smeared gauge links, are presented in what follows.

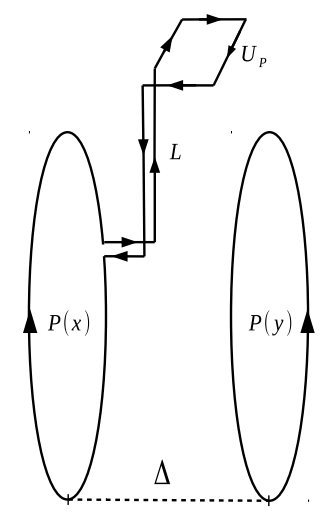

Figure 1: The connected correlator given in Eq. (2.1) (subtraction in $\rho_{P}^{\text {conn }}$ not explicitly drawn).

\section{Flux tubes on the lattice}

In order to explore the field configurations produced by a static $q \bar{q}$ pair, the following connected correlation function was considered:

$$
\rho_{P}^{\text {conn }}=\frac{\left\langle\operatorname{tr}\left(P(x) L U_{P} L^{\dagger}\right) \operatorname{tr} P(y)\right\rangle}{\langle\operatorname{tr}(P(x)) \operatorname{tr}(P(y))\rangle}-\frac{1}{3} \frac{\left\langle\operatorname{tr}(P(x)) \operatorname{tr}(P(y)) \operatorname{tr}\left(U_{P}\right)\right\rangle}{\langle\operatorname{tr}(P(x)) \operatorname{tr}(P(y))\rangle}
$$

The two Polyakov lines are separated by a distance $\Delta$. In the continuum limit we obtain the field strength tensor, defined as [15]

$$
F_{\mu v}(x)=\sqrt{\frac{\beta}{6}} \rho_{P}^{\text {conn }}(x) .
$$

Wilson action with periodic boundary conditions and the Cabibbo-Marinari algorithm [16] com- 

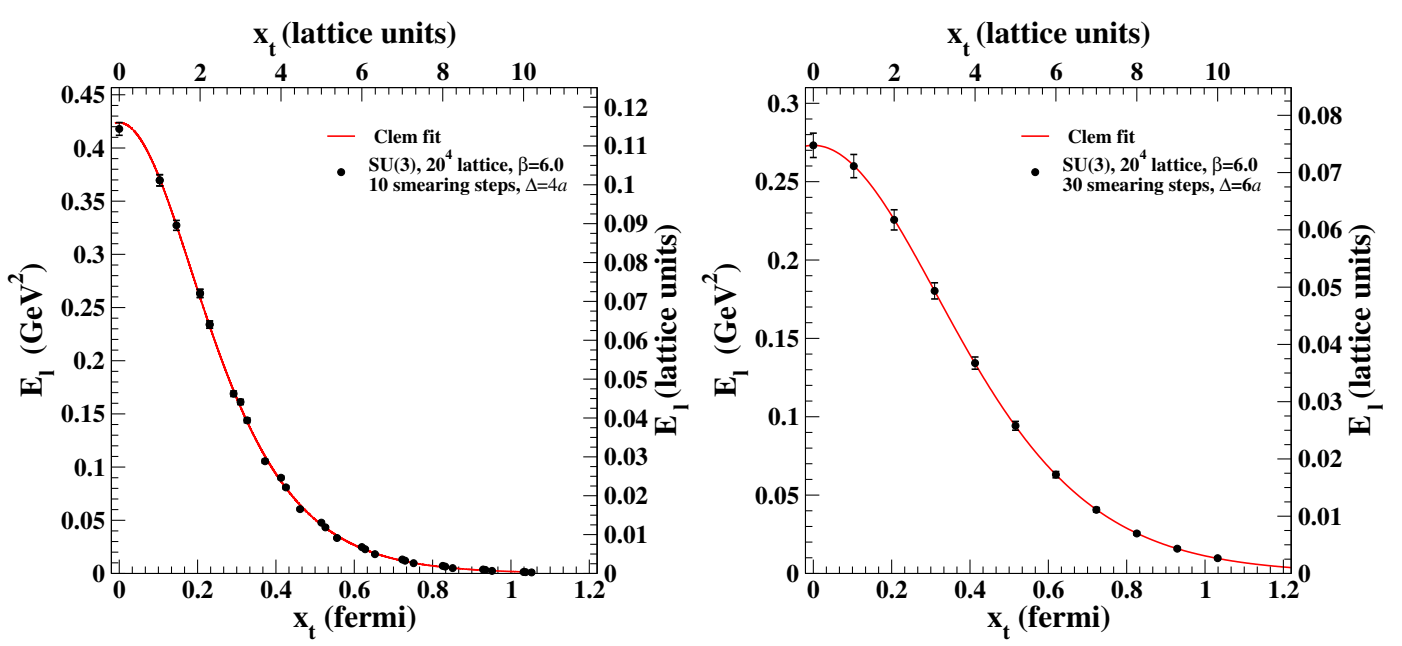

Figure 2: (Left) $E_{l}$ versus $x_{t}$, in lattice units and in physical units, at $\beta=6.0$ and for $\Delta=4 a$, after 10 smearing steps. Full line is the best fit using Eq. (1.5). (Right) The same, but for $\Delta=6 a$, after 30 smearing steps. The procedure to fix the physical scale is explained in Sect. 3.

bined with overrelaxation were used, and simulations were performed on $20^{4}$ lattices. We considered $\Delta=4 a, 6 a, 8 a$ ( $a$ is the lattice spacing), and four different values of the gauge coupling $\beta$ in the range $5.9 \div 6.1$. Measurements were taken every 10 updatings in order to reduce the autocorrelation time. The jackknife method was used for the error analysis. The smearing procedure as described in Ref. [13], with smearing parameter $\varepsilon=0.5$, was employed to reduce statistical errors. The flux tube is confirmed to be almost completely formed by the longitudinal chromoelectric field $E_{l}$, which is constant along the flux axis and decreases rapidly in the transverse direction $x_{t}$. To probe $E_{l}\left(x_{t}\right)$, the plaquette in Eq. (1.5) was placed in correspondence to the middle point (labeled by $x_{t}=0$ ) of the axis connecting the static sources and, then, moved along all possible transverse spatial directions to distances $x_{t}>0$ from that axis. We fitted our data to Eq. (1.5) and the result of the fit is shown in Fig. 2. To check rotational invariance, also noninteger distances were considered for $\Delta=4 a$, but, since the only effect of restricting the fit to integer distances was a reduction of the reduced chi-square, $\chi_{r}^{2}$, in order to have less time-consuming simulations, we performed measurements for integer transverse distances only, for all the other $\Delta$ values.

The fit of our data to Eq. (1.5) was realized for each smearing step in the interval $16 \div 50$. The parameters $\phi, \mu$, and $\lambda / \xi_{v}$ were extracted and the Ginzburg-Landau parameter $\kappa$ was evaluated through Eq. (1.6). Well-defined plateaux were found in the dependence of all parameters on the number of smearing steps (see table 1 for the $\beta=6.0$ and $\Delta=6 a$ case). In order to check for contamination effects, on $E_{l}$, due to the proximity of the static color sources, we varied $\Delta$. It was found that the $\mu$ and $\lambda / \xi_{v}$ values for $\Delta=4 a$ were systematically higher than for $\Delta=6 a, 8 a$, while, for all the parameters the values obtained for the $\Delta=6 a$ and $8 a$ were consistent within each other. The large statistical errors affecting our estimates for $\Delta=8 a$ led us to focus our analysis on the case $\Delta=6 a$ as a good compromise between the absence of contamination effects and a reasonable signal-to-noise ratio. Figure 3 shows the behavior of $\phi, \mu, \lambda / \xi_{v}$ and $\kappa$, for $\Delta=6 a$ and for different values of $\beta$, versus the number of smearing step. For $\beta \geq 6.0$, our estimate for the parameters seems to be independent of the number of smearing steps. 
Table 1: Summary of the fit values at $\beta=6.0$ for $\Delta=6 a$.

\begin{tabular}{cccccc}
\hline \hline Smearing & $\phi$ & $\mu$ & $\lambda / \xi_{v}$ & $\kappa$ & $\chi_{r}^{2}$ \\
\hline 16 & $6.191(141)$ & $0.621(79)$ & $0.309(95)$ & $0.213(91)$ & 0.018 \\
18 & $6.218(125)$ & $0.622(76)$ & $0.287(82)$ & $0.192(77)$ & 0.011 \\
20 & $6.227(109)$ & $0.617(68)$ & $0.277(72)$ & $0.183(66)$ & 0.010 \\
22 & $6.222(98)$ & $0.608(61)$ & $0.271(64)$ & $0.178(58)$ & 0.010 \\
24 & $6.207(88)$ & $0.597(55)$ & $0.269(58)$ & $0.176(53)$ & 0.011 \\
26 & $6.184(81)$ & $0.587(50)$ & $0.269(54)$ & $0.175(49)$ & 0.011 \\
28 & $6.155(75)$ & $0.576(47)$ & $0.269(51)$ & $0.176(46)$ & 0.011 \\
30 & $6.122(70)$ & $0.566(44)$ & $0.270(48)$ & $0.176(44)$ & 0.010 \\
32 & $6.087(66)$ & $0.557(41)$ & $0.271(46)$ & $0.177(42)$ & 0.009 \\
34 & $6.049(63)$ & $0.549(39)$ & $0.271(45)$ & $0.178(41)$ & 0.008 \\
36 & $6.011(60)$ & $0.541(37)$ & $0.272(43)$ & $0.179(40)$ & 0.007 \\
38 & $5.973(58)$ & $0.534(36)$ & $0.273(42)$ & $0.179(39)$ & 0.005 \\
40 & $5.935(56)$ & $0.527(35)$ & $0.274(42)$ & $0.180(38)$ & 0.004 \\
42 & $5.897(54)$ & $0.521(34)$ & $0.274(41)$ & $0.180(37)$ & 0.003 \\
44 & $5.859(53)$ & $0.515(33)$ & $0.275(40)$ & $0.181(37)$ & 0.003 \\
46 & $5.822(51)$ & $0.510(32)$ & $0.275(40)$ & $0.181(37)$ & 0.002 \\
48 & $5.786(50)$ & $0.505(31)$ & $0.276(39)$ & $0.182(36)$ & 0.002 \\
50 & $5.751(49)$ & $0.500(31)$ & $0.277(39)$ & $0.182(36)$ & 0.001 \\
\hline \hline
\end{tabular}

\section{Penetration and coherence lengths}

The estimation in physical units of the London penetration depth, $\lambda$, and the coherence length, $\xi$, is the final goal of our analysis. For this purpose it is, first of all, necessary to study the scaling of the plateau values of $a \mu$ with the string tension. To do this, we expressed the values of $a \mu$ in units of $\sqrt{\sigma}$, using the parameterization [17]:

$$
\begin{gathered}
\sqrt{\sigma}(g)=f_{\mathrm{SU}(3)}\left(g^{2}\right)\left[1+0.2731 \hat{a}^{2}(g)-0.01545 \hat{a}^{4}(g)+0.01975 \hat{a}^{6}(g)\right] / 0.01364, \\
\hat{a}(g)=\frac{f_{\mathrm{SU}(3)}\left(g^{2}\right)}{f_{\mathrm{SU}(3)}\left(g^{2}(\beta=6)\right)}, \beta=\frac{6}{g^{2}}, \quad 5.6 \leq \beta \leq 6.5,
\end{gathered}
$$

where

$$
f_{\mathrm{SU}(3)}\left(g^{2}\right)=\left(b_{0} g^{2}\right)^{-b_{1} / 2 b_{0}^{2}} \exp \left(-\frac{1}{2 b_{0} g^{2}}\right), \quad b_{0}=\frac{11}{(4 \pi)^{2}}, b_{1}=\frac{102}{(4 \pi)^{4}} .
$$

The use of the above parameterization allowed us also to compute and display, in Fig. 2, the transverse structure of $E_{l}\left(x_{t}\right)$ in physical units. Figure 4 (Left) shows the ratio $\mu / \sqrt{\sigma}$ versus $\beta$. For $\beta \geq 6.0, \mu$ scales according to the string tension. Likewise, the dimensionless Ginzburg-Landau parameter $\kappa$ scales in the same interval of $\beta$ (see Fig. 4 (Right)). Fitting, in both cases, the data in the scaling window with a constant we get

$$
\frac{\mu}{\sqrt{\sigma}}=2.684(97), \quad \kappa=0.178(21) .
$$

Assuming the standard value for the string tension, $\sqrt{\sigma}=420 \mathrm{MeV}$, from Eq. (3.3) we get

$$
\lambda=\frac{1}{\mu}=0.1750(63) \mathrm{fm}, \quad \xi=0.983(121) \mathrm{fm} .
$$

Our determinations appear to be in good agreement with the results in Ref. [11] which were obtained using the connected correlator built with the Wilson loop, and the cooling procedure. Type-I superconductivity of the SU(3) vacuum is confirmed and agreement is found also with Ref. [18]. 

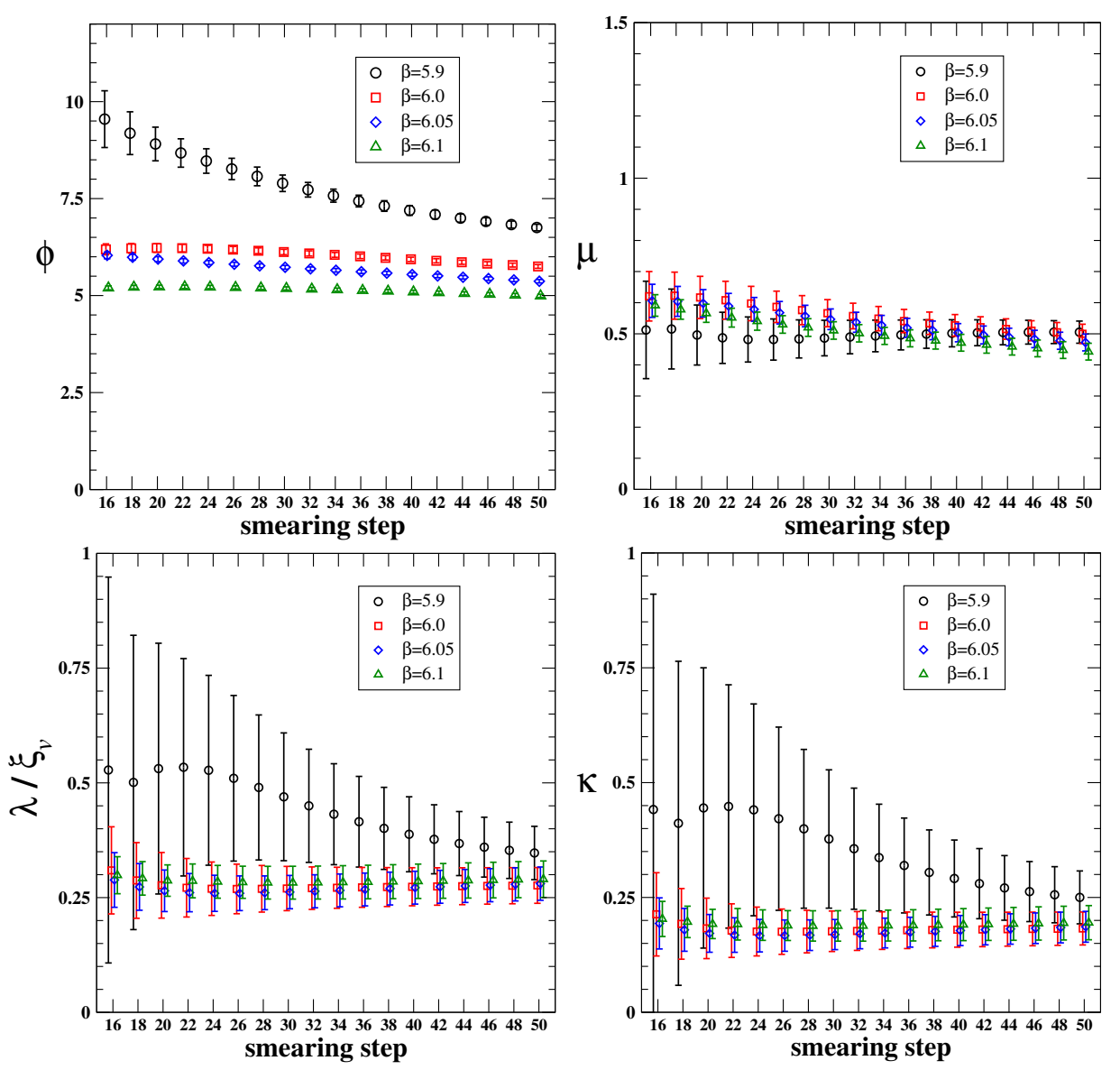

Figure 3: (Up left) $\phi$ versus the smearing step. (Up right) The same for the inverse of the penetration length $\mu$. (Down left) The same for $\lambda / \xi_{v}$. (Down right) The same for the Ginzburg-Landau parameter $\kappa$. In all cases $\Delta=6 a$, and in the last three figures data have been slightly shifted along the horizontal axis for the sake of readability.

\section{Acknowledgments}

Simulations have been performed on the $\mathrm{BC}^{2} \mathrm{~S}$ cluster in Bari. We also acknowledge support from the INFN - SUMA project.

\section{References}

[1] M. Bander, Phys. Rept. 75 (1981) 205; J. Greensite, Prog. Part. Nucl. Phys. 51 (2003) 1.

[2] M. Fukugita and T. Niuya, Phys. Lett. B 132 (1983) 374; J. E. Kiskis and K. Sparks, Phys. Rev. D 30 (1984) 1326; J. W. Flower and S. W. Otto, Phys. Lett. B 160 (1985) 128; J. Wosiek and R. W. Haymaker, Phys. Rev. D 36 (1987) 3297; V. Singh, D. A. Browne and R. W. Haymaker, Phys. Lett. B 306 (1993) 115; P. Cea and L. Cosmai, Nucl. Phys. Proc. Suppl. 30 (1993) 572; G. S. Bali, K. Schilling and C. Schlichter, Phys. Rev. D 51 (1995) 5165; R. W. Haymaker and T. Matsuki, Phys. Rev. D 75 (2007) 014501; A. D'Alessandro, M. D'Elia and L. Tagliacozzo, Nucl. Phys. B 774 (2007) 168.

[3] A. Di Giacomo, M. Maggiore and S. Olejnik, Nucl. Phys. B 347 (1990) 441.

[4] P. Cea and L. Cosmai, Nuovo Cim. A 107 (1994) 541; P. Cea and L. Cosmai, Nucl. Phys. Proc. Suppl. 34 (1994) 219; P. Cea and L. Cosmai, Phys. Lett. B 349 (1995) 343; P. Cea and L. Cosmai, Nucl. Phys. Proc. Suppl. 42 (1995) 225. 

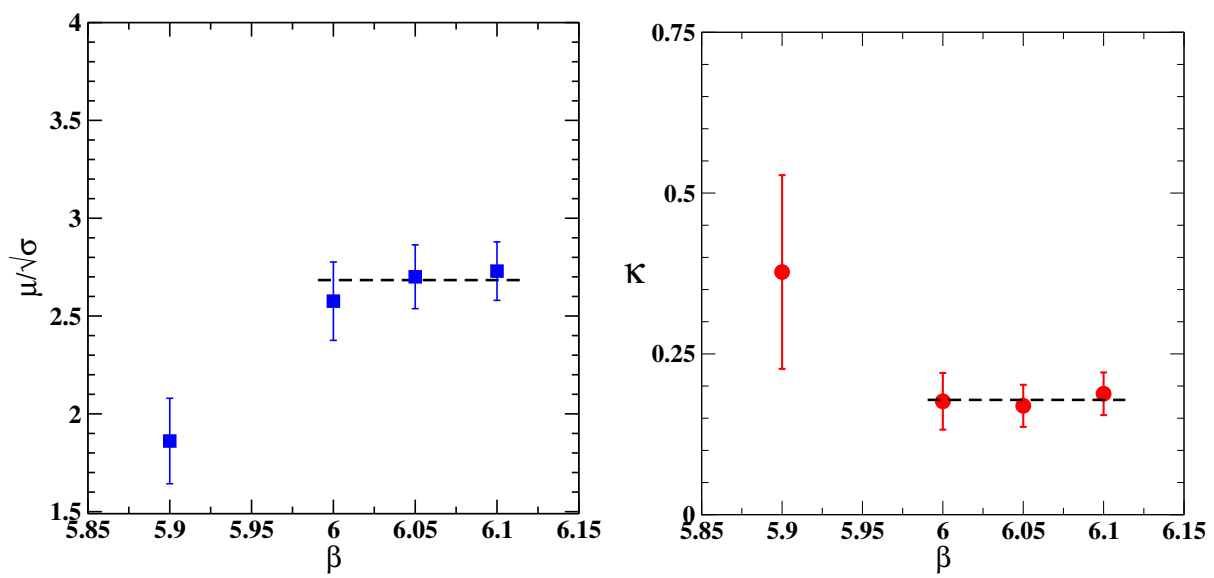

Figure 4: (Left) $\mu / \sqrt{\sigma}$ versus $\beta$ for $\Delta=6 a$. (Right) $\kappa$ versus $\beta$ for $\Delta=6 a$.

[5] S. Mandelstam, Phys. Rept. 23 (1976) 245; G. 't Hooft, EPS International Conference (1975); G. Ripka, (Lecture notes in physics. 639).

[6] H. Shiba and T. Suzuki, Phys. Lett. B 351 (1995) 519; N. Arasaki, S. Ejiri, S. i. Kitahara, Y. Matsubara and T. Suzuki, Phys. Lett. B 395 (1997) 275; P. Cea and L. Cosmai, Phys. Rev. D 62 (2000) 094510; P. Cea and L. Cosmai, JHEP 0111 (2001) 064; A. Di Giacomo, B. Lucini, L. Montesi and G. Paffuti, Phys. Rev. D 61 (2000) 034504; J. M. Carmona, M. D’Elia, A. Di Giacomo, B. Lucini and G. Paffuti, Phys. Rev. D 64 (2001) 114507; P. Cea, L. Cosmai and M. D'Elia, JHEP 0402 (2004) 018; A. D'Alessandro, M. D'Elia and E. V. Shuryak, Phys. Rev. D 81 (2010) 094501.

[7] P. Cea and L. Cosmai, Phys. Rev. D 52 (1995) 5152; M. S. Cardaci, P. Cea, L. Cosmai, R. Falcone and A. Papa, Phys. Rev. D 83 (2011) 014502.

[8] A. Di Giacomo, M. Maggiore and S. Olejnik, Phys. Lett. B 236 (1990) 199; D. S. Kuzmenko and Y. A. Simonov, Phys. Lett. B 494 (2000) 81; A. Di Giacomo, H. G. Dosch, V. I. Shevchenko and Y. A. Simonov, Phys. Rept. 372 (2002) 319.

[9] A. A. Abrikosov, Soviet Physics JETP (1957) 1174.

[10] T. Suzuki, Prog. Theor. Phys. 80 (1988) 929; S. Maedan, Y. Matsubara and T. Suzuki, Prog. Theor. Phys. 84 (1990) 130; V. Singh, D. A. Browne and R. W. Haymaker, Nucl. Phys. Proc. Suppl. 30 (1993) 568; Y. Matsubara, S. Ejiri and T. Suzuki, Nucl. Phys. Proc. Suppl. 34 (1994) 176; C. Schlichter, G. S. Bali and K. Schilling, Nucl. Phys. Proc. Suppl. 63 (1998) 519; G. S. Bali, C. Schlichter and K. Schilling, Prog. Theor. Phys. Suppl. 131 (1998) 645; K. Schilling, G. S. Bali and C. Schlichter, Nucl. Phys. Proc. Suppl. 73 (1999) 638; F. V. Gubarev, E. M. Ilgenfritz, M. I. Polikarpov and T. Suzuki, Phys. Lett. B 468 (1999) 134; Y. Koma, M. Koma, E. M. Ilgenfritz and T. Suzuki, Phys. Rev. D 68 (2003) 114504.

[11] P. Cea, L. Cosmai and A. Papa, Phys. Rev. D 86 (2012) 054501.

[12] J. R. Clem, J. Low Temp. Phys. 18 (1975) 427.

[13] M. Falcioni, M. L. Paciello, G. Parisi and B. Taglienti, Nucl. Phys. B 251 (1985) 624; M. Albanese et al. [APE Collaboration], Phys. Lett. B 192 (1987) 163.

[14] P. Cea, L. Cosmai, F. Cuteri and A. Papa, Phys. Rev. D 89 (2014) 094505.

[15] P. Skala, M. Faber and M. Zach, Nucl. Phys. B 494 (1997) 293.

[16] N. Cabibbo and E. Marinari, Phys. Lett. B 119 (1982) 387.

[17] R. G. Edwards, U. M. Heller and T. R. Klassen, Nucl. Phys. B 517 (1998) 377.

[18] A. Shibata, K. I. Kondo, S. Kato and T. Shinohara, Phys. Rev. D 87 (2013) 054011; N. Cardoso, M. Cardoso and P. Bicudo, Phys. Rev. D 88 (2013) 054504. 